\title{
High-fidelity linear optical quantum computing with polarization encoding
}

\author{
Federico M. Spedalieri, ${ }^{1, *}$ * Hwang Lee, ${ }^{1,2}$ and Jonathan P. Dowling ${ }^{1,2,3}$ \\ ${ }^{1}$ Jet Propulsion Laboratory, California Institute of Technology, \\ Mail Stop 126-347, 4800 Oak Grove Drive, Pasadena, California 91109-8099 \\ ${ }^{2}$ Hearne Institute for Theoretical Physics, Department of Physics and Astronomy, \\ Louisiana State University, Baton Rouge, Louisiana 70803-4001 \\ ${ }^{3}$ Institute for Quantum Studies, Department of Physics, \\ Texas AEM University, College Station, Texas 77843-4242
}

(Dated: April 18, 2018)

\begin{abstract}
We show that the KLM scheme [Knill, Laflamme and Milburn, Nature 409, 46] can be implemented using polarization encoding, thus reducing the number of path modes required by half. One of the main advantages of this new implementation is that it naturally incorporates a loss detection mechanism that makes the probability of a gate introducing a non-detected error, when non-ideal detectors are considered, dependent only on the detector dark-count rate and independent of its efficiency. Since very low dark-count rate detectors are currently available, a high-fidelity gate (probability of error of order $10^{-6}$ conditional on the gate being successful) can be implemented using polarization encoding. The detector efficiency determines the overall success probability of the gate but does not affect its fidelity. This can be applied to the efficient construction of optical cluster states with very high fidelity for quantum computing.
\end{abstract}

\section{INTRODUCTION}

The implementation of quantum computation using linear optical elements and measurements with postselection has attracted a great deal of attention since the seminal work by Knill, Laflamme, and Milburn [1]. In that paper the authors showed how the ideas of linear optical manipulation of photons, together with photodetection and postselection, can be combined with the concept of state teleportation through a quantum gate [2] to perform universal quantum computation. The price paid is that the two-qubit gates become non-deterministic. Whenever the gate fails, our qubit is measured in the computational basis with known outcome. A very important feature of this scheme is that gate failures are known, and this can be used to implement error correcting codes tailored to this particular situation.

In 1] the authors also showed that the success probability of the controlled-sign gate (CSIGN) can be made arbitrarily close to one by adding more ancilla modes. This result, combined with the existence of a threshold for quantum computation [3] implies that only a constant overhead is required to implement gates whose failure probability is below the threshold. A naive calculation shows that of the order of $10^{4}$ ancilla modes are required per two-qubit gate, which is difficult to achieve in practice. The situation can be improved by devising errorcorrecting codes that exploit characterisitics of the error model. This reduces the overhead required, but it still does not render the scheme easy to implement.

An approach that seems to be closer to being practical was proposed by Nielsen [4]. Instead of using the ideas of linear-optical quantum computing (LOQC) to

*Electronic address: Federico.Spedalieri@jpl.nasa.gov perform a quantum computation in the usual quantum circuit model, Nielsen proposed using the LOQC CSIGN gate to build optical cluster states that can then be used to do quantum computations as proposed by Raussendorf and Briegel [5]. The key point is that higher probabilities of gate failure can be tolerated while still being able to construct the required cluster state. Furthermore, even a gate that succeeds with arbitrarily small probability can be used to efficiently build a cluster state [6, 7]. This allows an LOQC gate with only a small number of ancilla modes to be sufficient for building the cluster state.

In its most basic form, the KLM scheme is extremely fragile against detectors errors. Failure to detect a photon introduces errors into the quantum state that are not detected. To minimize their effect on the quantum computation we need to have detectors with efficiencies above $99 \%$, which are far beyond from what is currently available. A modified scheme was also presented in Ref. [1], that would detect this photon loss at the price of requiring a more complicated entangled ancilla, with double the number of path modes required by the basic scheme. This would considerably complicate the implementation of this loss-detecting gate since the associated interferometer would be more difficult to control and stabilize. Nevertheless, such loss-detection mechanism is crucial even for the application of the basic KLM scheme to the construction of optical cluster states, since imperfect detectors can significantly affect the performance of the gate, introducing errors with probability of $30 \%$ and higher for currently available detectors, even when the gate is assumed to be successful.

The implementation of the KLM scheme using polarization encoding has two useful features. First, it requires half the number of path modes to implement the gates, which makes the associated interferometers easier to setup and control. And second, when using polarization encoding, the basic form of the KLM scheme already 
has a photon loss detection mechanism. The underlying reason for this feature emerges in a more natural way than for the modified dual-rail KLM scheme, and it is due to the conservation of the number of photons passing through a linear optical setup composed of mirrors, beam-splitters and phase-shifters. Even with the photon loss detection mechanism, the KLM scheme requires high-efficiency single-photon sources and high-efficiency detectors to apply the non-linear phase gate in the construction of an entangled ancilla with the required highfidelity. By implementing the scheme in the polarization basis, we can completely discard the requirement for high-efficiency detectors, if we replace the single-photon sources by high-fidelity Bell-pair sources. The errors introduced by the gate will then be due only to the darkcount rate of the detectors, which can be as low as $10^{-8}$ dark counts per gate. Thus, successful gates will also be high-fidelity gates, and they can be used to construct high-fidelity optical cluster states.

This paper is organized as follows. In Section II we present a detailed calculation of the KLM scheme using polarization encoding, including the application of the CSIGN gate and the generation of the required entangled ancillas. In Section III we discuss the effect of errors due to non-ideal detectors, both in the original KLM scheme and the one with polarization encoding, and show that the loss detection mechanism is crucial for the construction of a high-fidelity non-deterministic gate. In Section IV we discuss applications to the construction of optical cluster states and finally in Section V we present our conclusions.

\section{THE KLM SCHEME WITH POLARIZATION ENCODING}

One of the pillars on which the KLM scheme is based is the near-deterministic teleportation of the state of an optical mode using linear-optical elements, photodetectors, and an ancilla prepared in a particular entangled state. The success probability of this teleportation depends on the number of ancilla modes. For an ancilla state of $2 n$ modes, the probability of success is $\frac{n}{n+1}$. The teleportation procedure goes as follows. First, the mode to be teleported together with the first $n$ ancilla modes are sent through an optical device that performs a Fourier transform among the modes. This device can be constructed using beam-splitters, phase shifters, and mirrors. After the Fourier transform, we measure the number of photons present in each mode. For this step, number-resolving photodetectors are required. If the total number of photons measured is 0 or $n+1$, the gate failed and our qubit is measured in the computational basis. If $k$ photons are measured, with $0<k<n+1$, then the state of our qubit can be recovered by post-selecting mode $n+k$ of the ancilla and applying a phase shift that depends on the distribution of the photons measured among the first $n+1$ modes. The same can be accomplished with polarization encoding as we show below.

\section{A. Near-deterministic teleportation}

We will encode the state of our qubit (corresponding to an optical mode) into the polarization of the photon, so we will have $|0\rangle \rightarrow|H\rangle$ and $|1\rangle \rightarrow|V\rangle$. The state of our qubit will then be written as

$$
|\psi\rangle=\alpha|H\rangle+\beta|V\rangle .
$$

The ancilla state required to implement the teleportation has the same form as the state used in the original KLM paper with 0 replaced by $H$ and 1 by $V$. We will keep the same notation used in Ref. 1] and note this state by $\left|t_{n}\right\rangle$. Then we have

$$
\left|t_{n}\right\rangle=\frac{1}{\sqrt{n+1}} \sum_{j=0}^{n}|V\rangle^{j}|H\rangle^{n-j}|H\rangle^{j}|V\rangle^{n-j} .
$$

The main difference between this teleporting state and the one used in the original KLM is that (2) has exactly one photon per mode, for a total of $2 n$ photons. The analogous state in the original KLM scheme is

$$
\left|t_{n}\right\rangle_{K L M}=\frac{1}{\sqrt{n+1}} \sum_{j=0}^{n}|1\rangle^{j}|0\rangle^{n-j}|0\rangle^{j}|1\rangle^{n-j},
$$

that has only $n$ photons in $2 n$ modes. This difference will allow us to detect photodetector failure and hence minimize the errors introduced by the gate.

We will now give a detailed calculation of how the teleportation works. It is useful to write the states in terms of creation operators applied to the vacuum. We will call $a_{k}^{\dagger}$ the creation operator of a vertically polarized photon in mode $k$, and $b_{k}^{\dagger}$ the creation operator of a horizontally polarized photon in mode $k$. Then we have

$$
\begin{aligned}
a_{k}^{\dagger}|v a c\rangle & =|V\rangle_{k} \\
b_{k}^{\dagger}|v a c\rangle & =|H\rangle_{k},
\end{aligned}
$$

where $|v a c\rangle$ represents the vacuum state. We will write $|v a c\rangle_{1 \ldots n}$ to represent the vaccum state of modes 1 to $n$.

The Fourier transform applied to a set of $n+1$ modes, that for convenience we will call modes 0 to $n$, is given in terms of its action on the creation operators

$$
\begin{aligned}
& \hat{F}_{n}\left(a_{k}^{\dagger}\right)=\frac{1}{\sqrt{n+1}} \sum_{l_{k}=0}^{n} \omega^{k l_{k}} a_{l_{k}}^{\dagger} \\
& \hat{F}_{n}\left(b_{k}^{\dagger}\right)=\frac{1}{\sqrt{n+1}} \sum_{l_{k}=0}^{n} \omega^{k l_{k}} b_{l_{k}}^{\dagger}
\end{aligned}
$$

where $\omega=e^{i \frac{2 \pi}{n+1}}$. This Fourier transform can be implemented with linear optical elements [8]. One important point is that this operation does not mix the polarizations of the photon, which can be seen from Eqs. (5) in 
the fact that the creation operators for each polarization transform among themselves.

We can now rewrite the teleporting state $\left|t_{n}\right\rangle$ using the creation operators for horizontally and vertically polarized photons. So, we get

$$
\left|t_{n}\right\rangle=\frac{1}{\sqrt{n+1}} \sum_{j=0}^{n} a_{1}^{\dagger} \ldots a_{j}^{\dagger} b_{j+1}^{\dagger} \ldots b_{n}^{\dagger} b_{n+1}^{\dagger} \ldots b_{n+j}^{\dagger} a_{n+j+1}^{\dagger} \ldots a_{2 n}^{\dagger}|v a c\rangle_{1 \ldots 2 n}
$$

The teleportation trick starts by considering the joint state formed by our qubit in state (1) together with the state $\left|t_{n}\right\rangle$. Expanding this, we have

$$
|\psi\rangle\left|t_{n}\right\rangle=\frac{1}{\sqrt{n+1}} \sum_{j=0}^{n}\left\{\alpha b_{0}^{\dagger}\left(\prod_{k=1}^{j} a_{k}^{\dagger}\right)\left(\prod_{k=1}^{n} b_{j+k}^{\dagger}\right)\left(\prod_{k=1}^{n-j} a_{n+j+k}^{\dagger}\right)+\beta a_{0}^{\dagger}\left(\prod_{k=1}^{j} a_{k}^{\dagger}\right)\left(\prod_{k=1}^{n} b_{j+k}^{\dagger}\right)\left(\prod_{k=1}^{n-j} a_{n+j+k}^{\dagger}\right)\right\}|v a c\rangle_{0 \ldots 2 n} .
$$

This is a state of $2 n+1$ modes. Note that the difference between the two terms is, besides the values of $\alpha$ and $\beta$, that the first term has a creation operator for a horizontally polarized photon in mode 0 , while the second has a creation operator for a vertically polarized photon in that mode.

The next step is to apply the Fourier tansform to the first $n+1$ modes (i.e., modes 0 to $n$ ). Note that, since the two terms have different numbers of creation operators of each type $(H$ or $V$ ), and since the Fourier transform does not mix polarizations, the same will hold after the transformation is applied. The state obtained after the Fourier transform is

$$
\left(\frac{1}{\sqrt{n+1}}\right)^{n+2} \sum_{j=0}^{n}\left\{\sum_{0 \leq l_{0}, \ldots, l_{n} \leq n} \omega^{\sum_{k=0}^{n} k l_{k}}\left(\alpha b_{l_{0}}^{\dagger} a_{l_{1}}^{\dagger} \ldots a_{l_{j}}^{\dagger} b_{l_{j+1}}^{\dagger} \ldots b_{l_{n}}^{\dagger}+\beta a_{l_{0}}^{\dagger} a_{l_{1}}^{\dagger} \ldots a_{l_{j}}^{\dagger} b_{l_{j+1}}^{\dagger} \ldots b_{l_{n}}^{\dagger}\right)|v a c\rangle_{0 \ldots n}\right\} \underbrace{|H\rangle^{j}|V\rangle^{n-j}}_{\text {modes }(n+1, \ldots, 2 n)} \cdot
$$

Note that the $\alpha$ terms have $j$ of the $\mathrm{V}$-photons and $n-$ $j+1$ of the H-photons, while the $\beta$ terms have $j+1$ of the $\mathrm{V}$-photons and $n-j$ of the H-photons. This difference will be responsible for transfering the superposition in the state of mode 0 to one of the last $n$ modes of the ancilla.

Now the idea (following KLM) is to perform a measurement that collapses the state vector (8) to a certain value of $j$ for the $\alpha$ terms, and to $j-1$ for the $\beta$ terms. In the KLM scheme this is accomplished by measuring the number of photons in each of the $n+1$ output modes of the Fourier transform. Here however, that is not enough since there are two kinds of photons, so just measuring the number of photons present in each mode does not collapses the state vector (8) into the state we want. To solve this problem we just need to perform a stronger measurement that tells us not just how many photons are in one mode but how many of each polarization are present. This measurement can be easily implemented by sending the output of each mode through a polarizing beam-splitter (PBS), and then measuring the number of photons present in each of the two output ports of the
PBS. This requires the same number-resolving photodetectors used for the original KLM scheme, with the only difference that we now need twice as many.

Let us assume that we have performed this measurement, and we have obtained that in mode $j$, there are $r_{j}$ of the $\mathrm{V}$-photons and $h_{j}$ of the H-photons. Note that since there was one photon per mode in the first $n+1$ modes of (7), the total number of photons measured at the output of the Fourier transform must be $n+1$. This is the key feature that allows us to know when a detector fails to detect a photon. But now we want to know what is the state of the whole system after this projective measurement. First, let us consider the two simplest cases. If $\sum_{j=0}^{n} r_{j}=n+1$, then all the photons detected are $\mathrm{V}$-photons (i.e., $h_{j}=0, \forall j$ ). Looking at (8), we can see that the only term that has $n+1$ of the $\mathrm{V}$-photons in the first $n+1$ modes corresponds to the $\beta$ term with $j=n$. Any other term in (8) has at least one H-photon. Then the state corresponding to that measurement result is to be

$$
|V\rangle^{n+1}|H\rangle^{n}
$$


This correponds to a projective measurement of our qubit in the computational basis. The superposition is destroyed and the teleportation failed. The probability of obtaining this measurement result is $\frac{|\beta|^{2}}{(n+1)}$. Similarly, if we measure that $\sum_{j=0}^{n} r_{j}=0$, that means $\sum_{j=0}^{n} h_{j}=n+1$, and we can repeat the reasoning above replacing $\mathrm{V}$-photons by $\mathrm{H}$-photons. So again, the result is a projective measurement in the computational basis, which destroys the superposition. The probability of this event ocurring is $\frac{|\alpha|^{2}}{(n+1)}$, and so the total probability of failure of the teleportation is $\frac{1}{(n+1)}$ independent of the input state.

So let us now assume that $\sum_{j=0}^{n} r_{j} \neq n+1,0$, and write $\sum_{j=0}^{n} r_{j}=k$. Then we also have $\sum_{j=0}^{n} h_{j}=n-k+1$, since the total number of photons detected is always $n+1$. The state corresponding to that measurement result is

$$
\begin{aligned}
\left(\frac{1}{\sqrt{n+1}}\right)^{n+2} & \left\{\sum_{\mathcal{S}} \omega^{\sum_{p=0}^{n} p l_{p}} \alpha b_{l_{0}}^{\dagger} a_{l_{1}}^{\dagger} \ldots a_{l_{k}}^{\dagger} b_{l_{k+1}}^{\dagger} \ldots b_{l_{n}}^{\dagger}|v a c\rangle_{0 \ldots n}|H\rangle^{k}|V\rangle^{n-k}+\right. \\
+ & \left.\sum_{\mathcal{S}^{\prime}} \omega^{\sum_{p=0}^{n} p l_{p}} \beta a_{l_{0}}^{\dagger} a_{l_{1}}^{\dagger} \ldots a_{l_{k-1}}^{\dagger} b_{l_{k}}^{\dagger} \ldots b_{l_{n}}^{\dagger}|v a c\rangle_{0 \ldots n}|H\rangle^{k-1}|V\rangle^{n-k+1}\right\}
\end{aligned}
$$

with

$$
\begin{aligned}
& \mathcal{S}=\left\{\left(l_{0}, \ldots, l_{n}\right) /\left\{l_{1}, \ldots, l_{k}\right\} \text { contains the value } j, r_{j}\right. \text { times, and } \\
& \left.\left\{l_{0}, l_{k+1}, \ldots, l_{n}\right\} \text { contains the value } j, h_{j} \text { times, } j \in\{0, \ldots, n\}\right\} \\
& \mathcal{S}^{\prime}=\left\{\left(l_{0}, \ldots, l_{n}\right) /\left\{l_{0}, \ldots, l_{k-1}\right\} \text { contains the value } j, r_{j}\right. \text { times, and } \\
& \left.\left\{l_{k}, \ldots, l_{n}\right\} \text { contains the value } j, h_{j} \text { times, } j \in\{0, \ldots, n\}\right\} \text {. }
\end{aligned}
$$

By looking at the two sums in (10) we can see that these two terms have the same state for the first $n+1$ modes since they have the same number of $\mathrm{V}$-photons and $\mathrm{H}$-photons in each of the first $n+1$ modes (fixed by the result of the measurement). The only difference is given by the state of the last $n$ modes and by the factors introduced by the two sums

$$
\sum_{\mathcal{S}} \omega^{\sum_{p=0}^{n} p l_{p}} \text { and } \sum_{\mathcal{S}^{\prime}} \omega^{\sum_{p=0}^{n} p l_{p}} .
$$

Since the sums are over two different sets of $(n+1)$-tuples, it is not clear if this will change the relative weights in the superposition given by $\alpha$ and $\beta$. However, after a little algebra it can be shown that these two factors differ only by an overall phase. More precisely we have

$$
\sum_{\mathcal{S}^{\prime}} \omega^{\sum_{p=0}^{n} p l_{p}^{\prime}}=\sum_{\mathcal{S}} \omega^{\sum_{p=0}^{n} p l_{p}} \omega^{-\sum_{p=0}^{n} l_{p}}
$$

where $\omega=e^{\frac{2 \pi i}{n+1}}$ as defined earlier. By following the above calculation carefully, it is not hard to show that

$$
\sum_{p=0}^{n} l_{p}=\sum_{j=0}^{n} j\left(r_{j}+h_{j}\right) .
$$

It is worth noting that since $\left(r_{j}+h_{j}\right)$ is the total number of photons measured in mode $j$, this exponent has exactly the same form as the dephasing introduced by the teleportation procedure in the original KLM scheme. In summary we have that

$$
\sum_{\mathcal{S}^{\prime}} \omega^{\sum_{p=0}^{n} p l_{p}^{\prime}}=\omega^{-\sum_{j=0}^{n} j\left(r_{j}+h_{j}\right)} \sum_{\mathcal{S}} \omega^{\sum_{p=0}^{n} p l_{p}}
$$

Taking all of this into account we can rewrite the state (10) as

$|\Phi\rangle_{0 \ldots n}|H\rangle^{k-1}\left(\alpha|H\rangle+\beta \omega^{-\sum_{j=0}^{n} j\left(r_{j}+h_{j}\right)}|V\rangle\right)|V\rangle^{n-k+1}$,

where $|\Phi\rangle_{0 . \ldots n}$ is a normalized state of the first $n+1$ modes fixed by the result of the measurement. We can see that the superposition was teleported to the mode $n+k$, up to a known relative phase. But since we know exactly the value of that phase, we can get rid of it by using phase shifters, and then the final state becomes

$$
|\Phi\rangle_{0 \ldots n}|H\rangle^{k-1}(\alpha|H\rangle+\beta|V\rangle)|V\rangle^{n-k+1} .
$$

The last $n$ modes, with the exception of mode $n+k$ of course, are left in a known state and can be reused later. It is worth noting that the state (17) has exactly the same form as the state that we obtain in the original KLM scheme, if we replace all H's by zeros and all V's by ones. 


\section{B. Near-deterministic CSIGN}

The near-deterministic teleportation procedure described above can be combined with the idea of applying a quantum gate through teleportation to perform a near- deterministic CSIGN gate. To do this we will need a teleporting state of $4 n$ modes, which is nothing but two copies of the teleporting state $\left|t_{n}\right\rangle$ with CSIGN gates applied to the mode pairs $(n+k, 3 n+l), 1 \leq k, l \leq n$. This (unnormalized) state can be written as

$$
\left|t_{n}^{\prime}\right\rangle=\sum_{i, j=0}^{n}(-1)^{(n-j)(n-i)}|V\rangle^{j}|H\rangle^{n-j}|H\rangle^{j}|V\rangle^{n-j} \times|V\rangle^{i}|H\rangle^{n-i}|H\rangle^{i}|V\rangle^{n-i} .
$$

Note that this is a state of $4 n$ modes that has $4 n$ photons. To apply a CSIGN gate to two modes $A$ and $B$ we can proceed by teleporting the mode $A$ using the first $2 n$ modes of $\left|t_{n}^{\prime}\right\rangle$ and then teleporting mode $B$ using the last $2 n$ modes $\left|t_{n}^{\prime}\right\rangle$. Each teleporting step will proceed as before, requiring post-selection and phase correction, and failing independently with probability $\frac{1}{n+1}$. The calculation is essentially the one presented in the previous subsection applied twice, so we will not present it explicitly here.

\section{State preparation}

As described in Ref. [1] the KLM scheme for LOQC reduces to a state preparation problem: we need to be able to construct the state $\left|t_{n}^{\prime}\right\rangle$ using only linear optics and photodetection. In the original scheme with dual-rail encoding, we proceed as follows. From the state $|01\rangle|01\rangle$ of four optical modes (generated using a single photon source), we construct the state

$$
\frac{1}{2}(|01\rangle+|10\rangle)(|01\rangle+|10\rangle),
$$

by applying beam splitters to the mode pairs $(1,2)$ and $(3,4)$. Then we send modes 1 and 3 through a MachZender interferometer with a (non-deterministic) nonlinear sign-shift gate applied to each arm. This gate (which can be performed using linear optics and photodetection) applies the transformation

$$
\alpha_{0}|0\rangle+\alpha_{1}|1\rangle+\alpha_{2}|2\rangle \rightarrow \alpha_{0}|0\rangle+\alpha_{1}|1\rangle-\alpha_{2}|2\rangle .
$$

After the interferometer the state of the system is

$$
\left|t_{1}^{\prime}\right\rangle_{K L M}=\frac{1}{2}(|1010\rangle+|0110\rangle+|1001\rangle-|0101\rangle),
$$

which can be used to apply a CSIGN gate with probability $\frac{1}{4}$. To apply this gate with higher success probability we need to construct the states $\left|t_{n}^{\prime}\right\rangle$ with $n>1$. This is done by a recursive procedure that uses $\left|t_{p}^{\prime}\right\rangle$ to build $\left|t_{n}^{\prime}\right\rangle$, where $p<n$. By recycling these resources whenever a gate fails, it can be shown that the number of trials required to build $\left|t_{n}^{\prime}\right\rangle$ scales as $2^{O(\sqrt{n})}$.
Preparing the corresponding states with polarization encoding can be done much in the same way with some minor but essential modifications. First we should note that the analog of the beam-splitter transformation with dual-rail encoding, which takes the form

$$
|01\rangle \rightarrow \cos \theta|01\rangle+\sin \theta|10\rangle,
$$

cannot be implemented with linear optics if we are using polarization encoding, since in this case it takes the form

$$
|H V\rangle \rightarrow \cos \theta|H V\rangle+\sin \theta|V H\rangle,
$$

which is an entangling operation between two photons and cannot be implemented with linear optics alone. However, this operation can be applied nondeterministically if we are allowed to use the nondeterministic CSIGN. To see this, first note that the required trasformation is represented by the unitary matrix (in the basis $\{|H H\rangle,|H V\rangle,|V H\rangle,|V V\rangle\}$ )

$$
\left(\begin{array}{cccc}
1 & 0 & 0 & 0 \\
0 & \cos \theta & \sin \theta & 0 \\
0 & -\sin \theta & \cos \theta & 0 \\
0 & 0 & 0 & 1
\end{array}\right)
$$

and this matrix can be implemented by the quantum circuit All the one qubit operations can be applied deter-

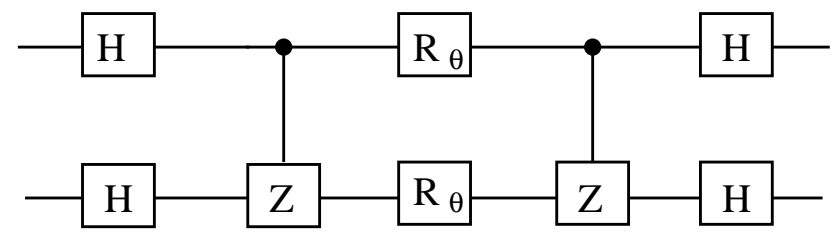

FIG. 1: Quantum circuit that applies the HV-beam splitter.

ministically, while the CSIGN (or C-Z) can be applied with some probability.

Let us assume for the moment that we can construct the state $\left|t_{1}^{\prime}\right\rangle$ with polarization encoding. Then we can generate the states $\left|t_{n}^{\prime}\right\rangle$ for $n>1$ following the same algorithm used in the original KLM scheme if we replace all beam-splitters by the circuit of Fig. 1] Following 
that procedure it is not hard to see that the only effect is that the number of non-deterministic gates required (roughly) doubles, but it remains linear on $n$. Thus the resource required to build the teleporting states $\left|t_{n}^{\prime}\right\rangle$ with polarization encoding are only polynomially bigger than the resources required in the original KLM scheme.

Finally, we need to show that we can construct the state

$\left|t_{1}^{\prime}\right\rangle=\frac{1}{2}(|V H V H\rangle+|H V V H\rangle+|V H H V\rangle-|H V H V\rangle)$,

using only linear optics and photodetection. It is interesting to note that this state is the same four-photon cluster state used by Walther et al. in their experimental demonstration of a one-way quantum computer [9]. First we note that we can deterministically transform a state with dual-rail encoding into a state with polarization encoding by using a polarizing beam-splitter (PBS), so we have

$$
\alpha|10\rangle+\beta|01\rangle \rightarrow \alpha|H\rangle+\beta|V\rangle .
$$

Now we can apply the quantum encoder introduced by Pittman et al. 10 plus polarization rotators to perform, with probability $\frac{1}{2}$, the operation

$$
\alpha|H\rangle+\beta|V\rangle \rightarrow \alpha|V H\rangle+\beta|H V\rangle .
$$

Composing these two transformations we can perform

$$
\alpha|10\rangle+\beta|01\rangle \rightarrow \alpha|V H\rangle+\beta|H V\rangle,
$$

with probability $\frac{1}{2}$. Now consider the state

$$
\frac{1}{2}(|1010\rangle+|0110\rangle+|1001\rangle-|0101\rangle) .
$$

This is nothing but the state $\left|t_{1}^{\prime}\right\rangle_{K L M}$ in the original KLM scheme, and we know it can be constructed with probability $\frac{1}{16}$ using linear optics and photodetection. By applying the transformation (28) to the mode pairs $(1,2)$ and $(3,4)$ in (29), we obtain the state (25) with probability $\frac{1}{64}$. This probability is not claimed to be optimal.

\section{ERRORS INTRODUCED BY REAL DETECTORS}

Both the original KLM scheme and its implementation with polarized photons described above assume all the detectors are perfect, i.e, they have unit quantum efficiency and zero dark counts. If we allow for real detectors and still require the scheme to apply the entangling gates with a probability of failure low enough such that error correction and fault tolerant design allows for arbitrarily long quantum computation, the required efficiencies turn out to be extremely high, compared to the presently available detectors. A naive estimation requires a detector efficiency higher than $99.99 \%$. A clever use of error correction, exploting the properties of the error model, may reduce this requirement. However, no significant improvement that would render the scheme viable with currently available detectors has been proposed so far.

On top of the high-efficiency requirement for the detectors, the scheme requires a rather large overhead in terms of extra ancilla modes. Again, a naive calculation of this overhead yields $10^{4}$ ancilla modes required per CSIGN gate. Exploiting the properties of the failure modes can reduce this requirement to about 50 ancilla modes per CSIGN gate. This is still a rather large number to be practical for an actual implementation.

A big step forward in reducing this required overhead was the proposal by Nielsen [4] to combine the techniques of the KLM scheme with the cluster-state model of quantum computation [5]. Rather than using the CSIGN gate for the actual computation, we can use it to construct a cluster state on which we can later perform our quantum computation. The advantage of this approach is that lower success probabilities can be tolerated with only a modest overhead on the time required to build the cluster state, thus reducing the extra ancilla modes to four.

However, this proposal cannot escape the requirement of very high efficiency detectors. The reason behind this is the fact that low-efficiency detectors will make the fidelity of the CSIGN gate low even when the gate is assumed to be successful. This will reduce the fidelity of the cluster state and require a larger state to accomodate for fault tolerance and error correction during the cluster state computation. At this point, using polarization encoding instead of dual-rail encoding shows a clear advantage. Polarization encoding allows for very high fidelity CSIGN gates, conditioned on gate success (at the price of a lower probability of success), even with currently available detectors. Since a cluster state can be efficiently constructed using gates with arbitrarily small success probability [6, 7], using polarization encoding allows for efficient construction of high-fidelity cluster states. In the remainder of this section, we will analyze in more detail the errors introduced by real detectors for both the original KLM scheme and the one with polarization encoding, and discuss the advantages of the latter.

\section{A. Errors in KLM}

We will model a real detector with two parameters: a quantum efficiency $\eta$ and a dark count rate $\lambda$. We will assume that the dark counts follow a Poisson distribution, so the probability of having $d$ dark counts during the measuring interval $\tau$ will be

$$
D(d)=e^{-\lambda \tau} \frac{(\lambda \tau)^{d}}{d !} .
$$

We can then write the conditional probability of the detector measuring $k$ photons when $l$ photons were present 
as 11.

$$
P_{D}(k \mid l)=\sum_{d=0}^{k} D(k-d)\left(\begin{array}{l}
l \\
d
\end{array}\right) \eta^{d}(1-\eta)^{l-d} .
$$

We now analyze the effects of real detectors on the implementation of the near-deterministic teleportation step, which is the basis of the whole scheme. Let us recall that, to perform that step, we need to measure the number of photons present in each of the first $n+1$ modes after applying a Fourier transform to them. The total number of photons measured, $k$, tells us whether the gate succeeded (if $k \neq 0, n+1$ ), and in that case to which one of the last $n$ modes of the ancilla was the state of our qubit teleported to. It is then clear that accurately determining the value of $k$ is essential for the success of the scheme. Imperfect detectors will degrade our ability to determine $k$.

The finite efficiency and dark-count rate of the detectors will sometimes produce a result $k^{\prime}$ for the total number of photons that is different from the actual number of photons present $k$, since some detectors may fail to detect one or more photons, while others will register dark counts. (For actual detectors, failure to detect a photon has a higher probability than dark counts, but we will keep our analysis general.) If $k^{\prime} \neq 0, n+1$, we will assume that the teleportation was successful and post-select the wrong output mode as the one carrying the state of our qubit. This wrong mode will be in either the $|0\rangle$ state or the $|1\rangle$ state, depending on whether $k^{\prime}<k$ or $k^{\prime}>k$. This is similar to the measurement error introduced by the teleportation failure associated with $k=0, n+1$ in the original scheme. The difference is that this new failure goes completely undetected. The gate is assumed to have succeeded when in fact it has introduced a measurement error that will propagate through the computation.

Another undetected error may occur when the number of non-detected photons is equal to the number of dark counts. In this case the total number of photons is correct, but their distribution among the first $n+1$ modes may have been changed. Since this distribution determines the phase correction that needs to be applied after a successful teleportation, a phase error may be introduced in the computation.

As we can appreciate, considering real detectors can modify the KLM scheme significantly. The main change is that the probability of a detected failure $p_{f}$ is no longer $1-p_{s}$, where $p_{s}$ is the probability of success, since we now have non-detected errors ocurring with some probability $p_{n d e}$. Also, the probability of a detected failure may no longer be independent of the input state. Actually, it depends on the input state whenever the probability of dark counts is different from the probability of photon non-detection, as is the case for currently available detectors. The root of this is our encoding of information in photon number, while the failure of the detectors is biased towards decreasing photons numbers (dark counts are usually negligible with respect to detection failure).
Using the conditional probabilities given by (31) we can compute the probabilities of detected failure $p_{f}$, and the probability of errors introduced by the teleportation conditioned on no detected errors, defined by $p_{e}=p_{n d e} /\left(1-p_{f}\right)$ for different values of detector efficiency and dark-count rates as a function of the number of ancilla modes $n$. In particular, we considered the parameters corresponding to the number-resolving photodetector demonstrated by Miller et al. [12]. In that work a $20 \%$ quantum efficiency was reported, with dark counts of the order of $10^{-7}$, when considering a measuring time $\tau \sim 100 \mu s$. It was also reported that this scheme could be improved to achieve an efficiency of $80 \%$. Using this value, we plotted the different probabilities for $n=2,3,4$. As we can see from Fig. 2] the probability of a detected

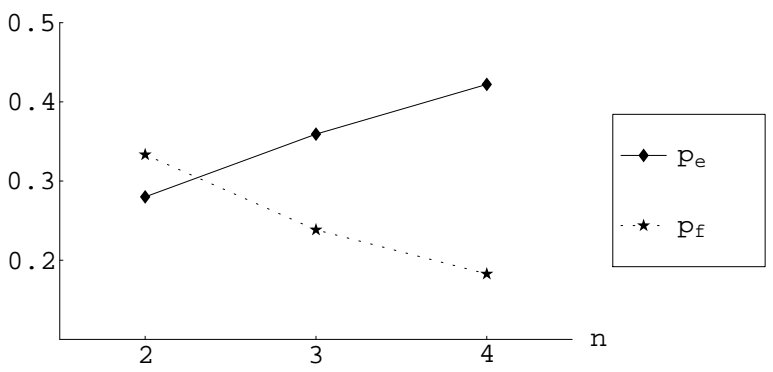

FIG. 2: Probability of detected failure $p_{f}$ and probability of error $p_{e}$ introduced by a "successful" gate in the KLM scheme $\left(\eta=0.8, \lambda \tau \sim 10^{-7}\right)$.

failure still decreases with $n$. However, the probability of an error being introduced by the gate when no failure is detected increases with $n$. Furthermore, this error is rather high $(\sim 27 \%)$ even for $n$ as small as 2. For $n=4$ this error is greater than $40 \%$. This can be easily understood since increasing $n$ increases the number of non-detected failure modes. From this point of view, increasing $n$ is actually counterproductive.

\section{B. Errors with polarization encoding}

The effect of errors introduced by real detectors when we use polarization encoding is remarkably different. As discussed before, the role of the total number of photons is played by the total number of vertically polarized photons. Since both the teleporting state and the input qubit have exactly one photon per mode, the total number of photons (both vertically and horizontally polarized) measured after the Fourier transform must be $n+1$. If this number is different from $n+1$ then we know for sure that some of the detectors failed, and we have lost the information about which mode the input qubit was teleported to. This case should be considered as a detected failure of the gate. Note that the most common error for real detectors (failure to detect a photon) will be recognized, while it would have gone undetected in the original KLM 
scheme.

Even if we use polarization encoding, there would be undetected errors occurring when we assume the gate has succeeded. These errors will require the same number of detector failures to measure a photon and registered dark counts. Depending on which detectors register these failures, we may choose the wrong output mode (i.e., we have the wrong information about the total number of vertically polarized photons present), or we may introduce a phase error (total number of V-photons is correct but their distribution among modes is not). The key point is that the probability of these type of errors is dramatically reduced because of the low probability of dark counts in currently available detectors. Using the same detector parameters discussed above, we computed the probability of detected failure $p_{f}$ for the teleportation step (see Fig. 3). We can see that the probability of a detected

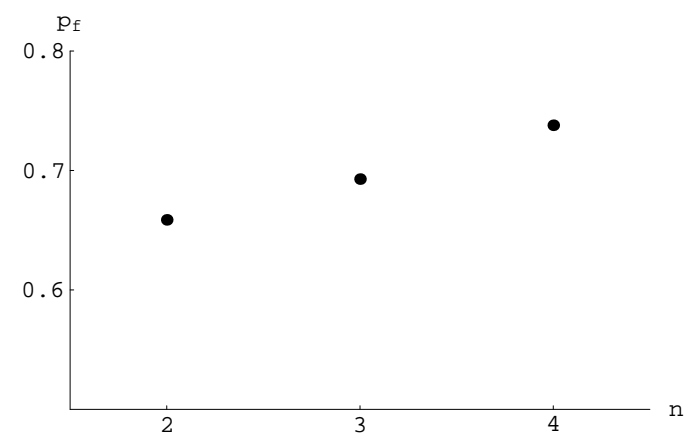

FIG. 3: Probability of detected failure $p_{f}$ using polarization encoding $\left(\eta=0.8, \lambda \tau \sim 10^{-7}\right)$.

failure is greater than for the KLM scheme $(66 \%$ compared to $33 \%$ for $n=2$ ). Furthermore, this probability increases with $n$ instead of decreasing. This can be easily understood. First, the probability of a detected failure depends essentially on the efficiency of the detectors. When using polarization encoding, we require double the number of detectors than for the KLM scheme, thus it is not surprising that our gate has a higher probability of failing. The scaling with $n$ may not have been foreseen but should not be unexpected, since now the failure probability includes errors derived from detector failures that are more abundant for higher values of $n$, as the number of detectors required is $2(n+1)$.

We have also computed the probability of error conditioned on no detected failure, $p_{e}=p_{n d e} /\left(1-p_{f}\right)$, with $p_{\text {nde }}$ the probability of a nondetected error. The results are shown in Fig. 4. This error probability is several orders of magnitude lower than the corresponding one for the KLM scheme using the same detector parameters. Its order of magnitude is given roughly by the order of magnitude of the dark-count probability since the most likely error corresponds to one detector failing to register a photon and another detector registering a dark count (events with higher number of dark counts are strongly suppressed). Lowering this probability can

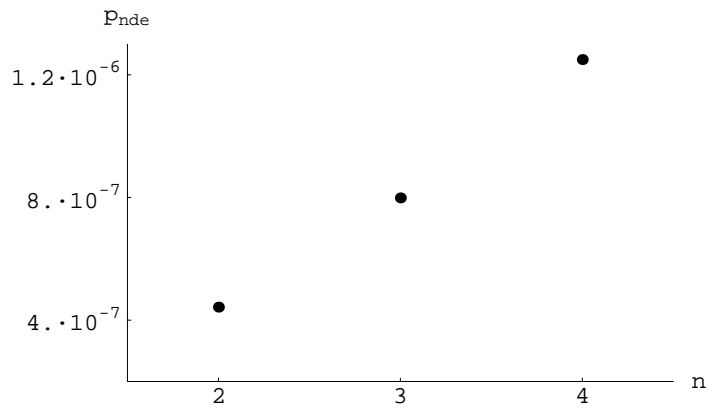

FIG. 4: Probability of error $p_{e}$ introduced by the gate when no failure was detected and using polarization encoding $(\eta=$ $0.8, \lambda \tau \sim 10^{-7}$ ).

be achieved by reducing the dark count rate, independent of the detector efficiency. We still have the same behavior with increasing $n$ that is related to the higher number of events associated with errors as the number of detectors required grows.

\section{APPLICATION TO CLUSTER STATE QUANTUM COMPUTING}

As we discussed before, coupling the KLM scheme with the cluster state model of quantum computation greatly reduces the number of ancilla modes required. This, together with the existence of protocols to efficiently build these cluster states with non-deterministic gates, makes this approach very appealing. For optical cluster states however, other issues need to be addressed. For the cluster state approach to be successful we need to be able to deal with the inevitable errors that will occur during its construction and during the computation itself. Since the computation proceeds by performing single qubit measurements while the quantum information is propagated along the cluster by quantum correlations, an efficient measurement procedure is required. This is not that simple in an optical implementation since currently available photodetectors do not have very high quantum efficiency. A good detector may have an efficiency of $80 \%$, which means that on average one in five photons of the cluster will not be detected. This rate of loss of cluster qubits can make quantum computing impossible. An incremental encoding was proposed by Gilchrist et al. [13] to make the computation fault tolerant to photon loss. Another idea recently proposed by Varnava et al. 14], exploits the fact that the cluster state is an eigenstate of a set of stabilizer operators to infer the results of measurements on photons that fail to be detected by the photodetectors. This scheme allows us to perform the required measurements for a quantum computation provided the efficiency of the detectors is above $50 \%$ (which are currently available).

Errors can also be introduced during the construction of the cluster, which in turn may introduce non- 
Markovian errors during the computation. Fortunately, as in the case of the quantum circuit model of quantum computing, there is an error threshold below which a fault tolerant design allows us to perform an arbitrarily long computation, although the value of this threshold is not yet known for the cluster state model [15, 16]. However, it is not expected to be significantly smaller than the threshold for the quantum circuit model since we can recast the cluster state approach as a quantum circuit. For example, we can consider the qubits in the cluster to be a quantum registry initialized in a particular product state, the CSIGN gates required to build the cluster can be regarded as the gates in the circuit model, and the measurements that implement the computation on the cluster can be considered as part of the readout measurements in the circuit model. Assuming perfect measurements, this shows that a CSIGN gate with error probability below the quantum circuit threshold should be enough to construct a cluster state with fidelity high enough to allow quantum computing in the cluster state model. The typical value quoted for the error threshold in the quantum circuit model is around $10^{-4}$, which corresponds to a general error model. A higher threshold might be possible if we take advantage of the properties of the error model associated with a particular implementation.

Thus emerges the greatest advantage of using polarization encoding instead of dual-rail encoding. Dual-rail encoding has a very high probability of non-detected errors being introduced when applying a CSIGN gate, and hence when constructing a cluster state using Nielsen's approach, unless very high efficiency detectors are used. On the other hand, polarization encoding allows us to reduce this non-detected errors independently of the efficiency of the detectors. The only requirement is that the dark-count rate of the detectors be sufficiently small, which is actually the case for currently available detectors. For example, a number-resolving photodetector was reported in Ref. [12] with a $20 \%$ efficiency and dark-count rate of the order of $10^{-9}$ for a measuring time of $1 \mu \mathrm{sec}$. Another number-resolving detector with an efficiency of $88 \%$ has been reported in Ref. [17]. Although this detector was reported to be essentially noise-free, no measured value of the dark-count rate was given.

There are several recipes to efficiently build a cluster state using non-deterministic gates [6, 7], and any of these approaches can be used to build an optical cluster state using the techniques described in this paper. The one specific advantage that we would like to exploit is the low probability of non-detected errors when using polarization encoding. As we saw in Fig. 团 this probability increases with the number ancilla modes used in the application of the CSIGN gate, so the smallest entangled ancilla state required (four photons in four modes) is actually the one that minimizes the error probability.
Using the smallest entangled ancilla state given by

$\left|t_{1}^{\prime}\right\rangle=\frac{1}{2}(|V H V H\rangle+|H V V H\rangle+|V H H V\rangle-|H V H V\rangle)$,

has some other advantages. In Fig. [5 we can see the setup required to implement the CSIGN gate. One advantage is

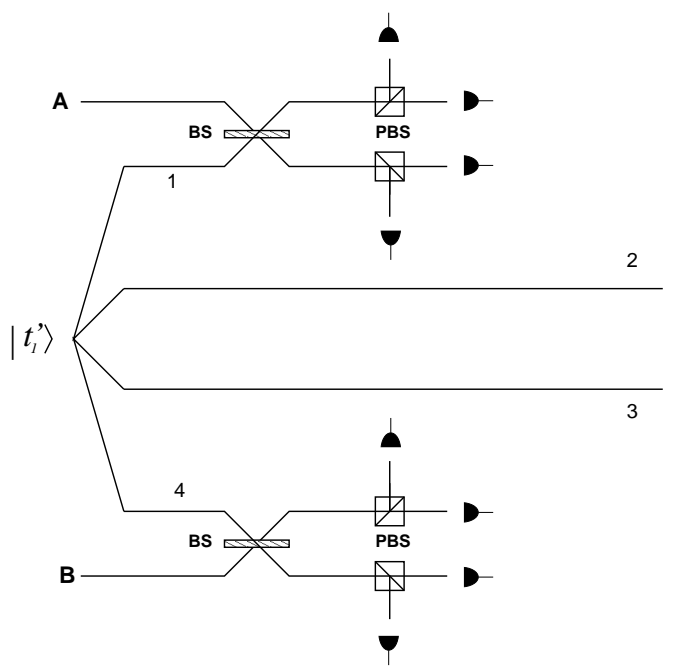

FIG. 5: Optical setup required to implement a CSIGN between two photons using a four photon entangled ancilla state. The two input modes $\mathrm{A}$ and $\mathrm{B}$ are combined through beamsplitters with modes 1 and 4 of the entangled ancilla $\left|t_{1}^{\prime}\right\rangle$, respectively. After the beam-splitters, the number of vertically and horizontally polarized photons in each mode is measured. When the gate succeeds, modes 2 and 3 carry the state of the input modes with a CSIGN gate applied to them, up to phase shifts.

that we only need to apply the optical Fourier transform to a pair of modes and that can be accomplished with a single balanced beam-splitter. We do not need to apply the more complicated interferometer required when more than two modes are input to the Fourier transform, which is experimentally very challenging. Also, since after the measurements only two modes are left, we do not have to physically post-select the output modes and only a phase shift may need to be applied to them. These two properties could be very useful if we want to integrate this gate into an optical chip, since no complex interferometers are involved and the output modes are fixed.

Furthermore, the success or failure of the gate is heralded by the number of vertically and horizontally polarized photons detected, while the total number of photons that must be detected is fixed and equal to four. Each step of the teleportation succeeds only when one vertically polarized photon and one horizontally polarized photon are detected, which in the setup presented in Fig. 5 means that two independent detectors must fire. This feature allows us to implement the same gate with detectors that are not number-resolving. Since a success corresponds to two independent detectors clicking, we do not need to know how many photons each detector 
measured. Again, the only possible errors introduced by the detectors malfunctioning are related to dark counts, which have an extremely low probability.

Besides the errors introduced by the detectors, the fidelity of this CSIGN gate can be affected by errors in the entangled ancilla state. If the state we use is not exactly the state (32), the gate applied will not be exactly a CSIGN gate, even when the measurements tell us it was successful. Thus, to assure the high fidelity of the gate we need to require the fidelity of the entangled ancilla with respect to the ideal state given by (32) to be high. Since we want the gate to work with fidelity at least $1-10^{-4}$ (to be below the error threshold) we need the fidelity between the two states to be of the same order. In Section II.C. we showed that this state can be constructed with linear optical elements and photodetectors, but this construction is based in the nonlinear sign (NS) gate that is the basis of the KLM scheme, and the fidelity of the NS gate is very fragile against detector inefficiencies because it operates in the photon number basis.

Nevertheless, we can non-deterministically construct a high efficiency copy of (32) with linear optics and nonideal detectors if we have access to high-fidelity polarization Bell pairs. We first combine two Bell pairs to form a GHZ state that might not have high-efficiency. To do this, we mix one mode from each Bell pair on a balanced beam-splitter and measure the number of photons in one of the outgoing modes irrespective to their polarization, as seen in Fig. 6] If only one photon is measured by the

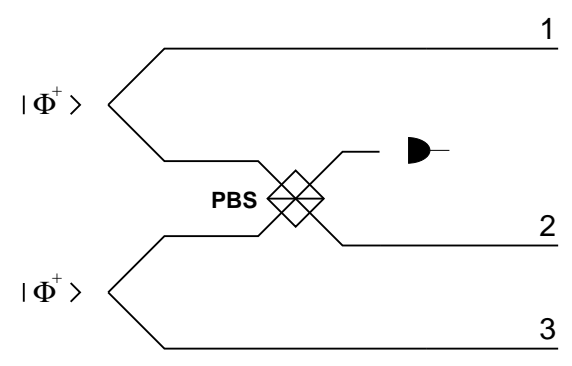

FIG. 6: Generation of a polarization GHZ state from two Bell pairs using a polarization beam-splitter and photodetection. A detector failure will introduce an error only in mode 2 , which in that case will contain no photons.

detector, the state of the remaining three modes is a GHZ state in the polarization basis. If that number is zero or two, the procedure is aborted. If non-ideal detectors are used an error may be introduced when two photons arrive at the detector but only one is registered due to its non-unit efficiency. This operation is very similar to the type-I fusion introduced by Browne and Rudolph [18]. The important point is that even when this error occurs, the only output mode affected is the one coming out of the beam-splitter that will then have no photons. The other two output modes will still have one photon each.

The second step is to take two copies of this GHZ state and combine the two modes that may have an error (i.e., no photon) using polarization rotators, polarization beam-splitters and phase shifters, and then measure the number of photons in each mode irrespective to their polarization, as seen in Fig. (7). If there were no errors in

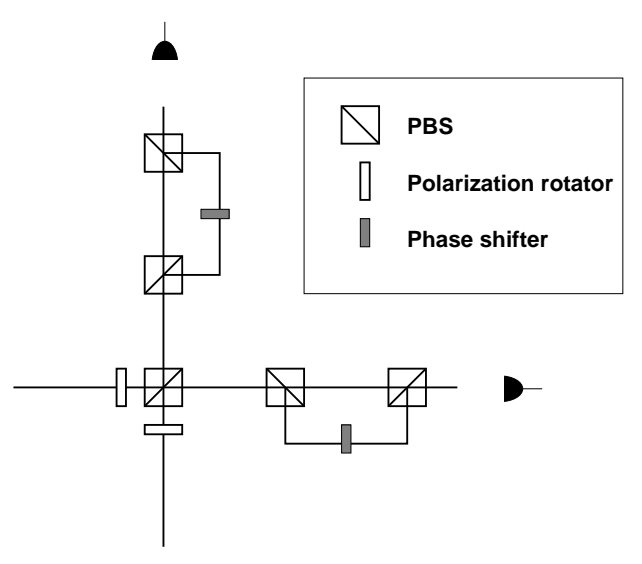

FIG. 7: The two modes of two pairs of GHZ states constructed according to the scheme in Fig. [6 that may have an error, are sent through the setup shown above. The polarization beam-splitters reflect vertically polarized photons. The operation is successful when exactly one photon is registered by each detector. Then, the state of the remaining four modes of the the two GHZ pairs can be transformed into $\left|t_{1}^{\prime}\right\rangle$ using phase-shifters and polarization rotators.

the construction of the two GHZ states, we expect exactly two photons to be detected. If we detect less than two photons, the GHZ states had errors and the procedure is aborted. If we detect two photons in one of the detectors (and zero in the other one), the output state is equivalent to two Bell pairs that can be reused to generate more GHZ states. If we detect one photon in each mode, the state of the remaining output modes is given by

$$
\begin{aligned}
\frac{1}{2}\left(e^{i \frac{\pi}{4}}|H H H H\rangle+e^{-i \frac{\pi}{4}}|H H V V\rangle+\right. \\
\left.+e^{-i \frac{\pi}{4}}|V V H H\rangle+e^{i \frac{\pi}{4}}|V V V V\rangle\right) .
\end{aligned}
$$

By applying polarization rotators and polarizationdependent phase-shifters, we can transform this state into (32). The key feature of this construction is that even though an error may be introduced in the first step by a non-ideal detector, the effect of this error will be restricted to a photon missing in only a pair of modes, and it can be detected when these two modes are mixed and the total number of photons is measured 19]. Then, an error in the final cluster state can only be related to the occurrence of a dark count in one of the detectors, and for currently available detectors the probability of such event is below $10^{-6}$. As long as the fidelity of the initial bell pairs is high enough, this procedure generates a four-photon high-fidelity cluster state that can be used to apply a high-fidelity CSIGN gate. 


\section{CONCLUSIONS}

In this paper we have shown that the KLM techniques can be extended to the case of polarization encoding. This implementation has two main advantages. Firstly, it requires half the number of path modes when compared to the usual dual-rail encoding. This can be very helpful in an actual implementation, since a higher number of path modes makes it more difficult to control and stabilize the interferometric setup required to implement the gates. And secondly, when polarization encoding is used, the analogous to the basic KLM scheme already has a photon loss detection mechanism. This is due to the fact that with this encoding we have one photon per path mode, instead of the one photon per two path modes of dual-rail encoding. Thus, when applying the nondeterministic gates using photodetection, we know the total number of photons that are expected, and the information about the operation of the gate is carried by the distribution of horizontally and vertically polarized photons.

The original KLM scheme can be modified to also include a photon loss detection mechanism. This requires a more complicated entangled ancilla that has double the number of path modes. Polarization encoding not only reduces this number by half, but also incorporates the loss mechanism in a natural way that makes it easier to understand how it works. It is just a consequence of the fact that the total photon number is conserved by any linear-optical device. The construction of the entangled ancilla can proceed much in the same fashion as in the KLM case, although the number of nondeterministic steps required roughly doubles. This is due to the fact that the polarization entangled ancilla have double the number of photons.

We have also studied numerically the effects of detector efficiency on the basic KLM scheme and showed how important having a loss detection mechanism is. Without it, even when the gate is assumed to be successful the probability of errors can be as high as 30\%, and furthermore it increases with the number of ancilla modes. Even though for perfect detectors the probability of success of the gate increases with the number of ancillas, with imperfect detectors the probability of the gate introducing no errors actually increases with the number of ancilla modes. This shows that even for applications in which the success probability of the gate is not required to be high (as in the construction of cluster states following Nielsen's proposal), either loss detection or the use of high-efficiency detectors are crucial for the success of the scheme.

Another interesting feature is that if the smallest entangled ancilla is used, which is a four-mode entangled state, the implementation of the CSIGN gate becomes simpler. On one hand it does not require numberresolving photodetectors, and on the other hand only a beam-splitter is required to mix the input modes with the entangled ancila, instead of the very complex interferometer required by the general Fourier transformation in the KLM implementation. Incidentally, it is worth noting that this mixing with a beam splitter is very similar to the Type-II fusion operation introduced by Browne and Rudolph [18], which can also be used to grow cluster states. This operation also has the nice feature of its fidelity being independent of the detector efficiency (although this point was not mentioned by the authors.) This simplified implementation of the gate, together with the fact that the output modes need not be physically post-selected when the gate is successful, makes it very appealing for integration into an optical chip.

It is important to note that even with the loss detection mechanism, the KLM scheme requires high-efficiency detectors and high-fidelity single-photon sources in order to apply the nonlinear sign gate that is the basis of the scheme. Here we have presented and alternative approach that allows us to do away with high-efficiency detector and single-photon source requirements, provided we have access to a high-fidelity polarization-entangled Bell pair source. This gives us another possible path to the implementation of LOQC with low errors using currently availabe detectors.

\section{ACKNOWLEDGMENTS}

Part of this work was carried out at the Jet Propulsion Laboratory, California Institute of Technology, under a contract with the National Aeronautics and Space Administration (NASA). FMS acknowledges support from the National Research Council and NASA Code Y. JPD and HL acknowledge support from the Hearne Foundation, the National Security Agency, the Advanced Research and Development Activity and the Army Research Office.
[1] E. Knill, R. Laflamme, and G. J. Milburn, Nature 409, 46 (2001).

[2] D. Gottesman and I. Chuang, Nature 402, 390 (1999).

[3] M. N. Nielsen and I. L. Chuang, Quantum computation and quantum information (Cambridge University Press, Cambridge, 2000).

[4] M. A. Nielsen, Phys. Rev. Lett. 93, 040503 (2004).

[5] R. Raussendorf and H. J. Briegel, Phys. Rev. Lett. 86,
5188 (2001).

[6] L.-M. Duan and R. Raussendorf, quant-ph/0502120 (unpublished).

[7] S. D. Barrett and P. Kok, Phys. Rev. A 71, 060310 (2005).

[8] M. Reck, A. Zeilinger, H. J. Bernstein, and P. Bertani, Phys. Rev. Lett. 73, 58 (1994).

[9] P. Walther et al., Nature 434, 169 (2005). 
[10] T. Pittman, B. Jacobs, and J. Franson, Phys. Rev. A 64, 062311 (2001).

[11] H. Lee et al., Journal of Modern Optics 51, 1517 (2004).

[12] A. J. Miller, A. W. Nam, J. M. Martinis, and A. V. Sergienko, Applied Physics Letters 83, 791 (2003).

[13] A. Gilchrist, A. J. F. Hayes, and T. C. Ralph, quantph/0505125 (unpublished).

[14] M. Varnava, D. E. Browne, and T. Rudolph, quantph/0507036 (unpublished).

[15] M. A. Nielsen and C. M. Dawson, quant-ph/0405134 (un- published).

[16] P. Aliferis and D. W. Leung, quant-ph/0503130 (unpublished).

[17] D. Rosenberg, A. E. Lita, A. J. Miller, and S. W. Nam, Phys. Rev. A 71, 061803 (2005).

[18] D. E. Browne and T. Rudolph, Phys. Rev. Lett. 95, 010501 (2005)

[19] We are grateful to T. Ralph for enlightening us about this point. 